\title{
Éditorial
}

\section{Notre journal change}

Je viens ici vous expliquer les changements importants qui vont intervenir dès le premier numéro de 2005.

Par le passé, vous avez vu apparaître des nouvelles rubriques, Internetécl@ire en est une et elle semble vous satisfaire. Il y aura encore des nouveautés, comme la rubrique «Débats », courant 2005, mais aujourd'hui le point majeur est la prochaine consultation de l'intégralité des articles de la revue, au format PDF, sur le site de la revue (http://www.edpsciences.org/radio/).

En effet, à la demande de notre président, la revue sera dorénavant consultable en ligne pour les abonnés et les membres de la société ; notre secrétaire général vous enverra le mode d'accès dans son bulletin électronique. C'est une excellente nouvelle et nous allons profiter de cette opportunité pour encore améliorer notre revue et son implantation internationale.

Qu'allons-nous faire?

Dès le premier numéro de 2005 , la revue sera donc consultable sur Internet et référencée dans les grandes bases de données. Elle l'était déjà dans certaines, nous allons travailler pour accroître leur nombre et par conséquent, la lisibilité de la revue.

À cette occasion, plusieurs nouveautés seront mises en place :

- bien qu'il soit possible de faire une recherche par mot-clef dans le texte, nous demanderons à nos auteurs, dès aujourd'hui, de joindre à leur résumé plusieurs mots-clefs qui seront mis en exergue dans l'en-tête de l'article. Une liste leur sera proposée, très proche des grands standards américains (Health Physics par exemple);

- les bibliographies de chaque article seront présentées avec des liens : ainsi, grâce notamment au système «CrossRef» (système auquel adhère notre éditeur), le lecteur pourra directement avoir accès à la revue citée, à condition que celle-ci figure sur la liste des revues internationales indexées. D'autres bases d'indexation en dehors de CrossRef seront également utilisées, comme par exemple Medline. À l'inverse nos articles seront consultables directement s’ils sont cités par ces mêmes revues. Les auteurs seront donc encouragés à internationaliser leurs références et le Comité de rédaction y veillera ;

- nous allons également procéder progressivement à l'archivage de la revue et installer sur le site tous les articles (environ 180) depuis 1997. Nous verrons ensuite, selon le succès de cette opération, s'il est nécessaire de remonter jusqu'à la création de la revue. 
ÉDITORIAL

De nouvelles instructions aux auteurs seront donc publiées dans le dernier numéro 2004.

Cette étape, qui n'est pas anodine, devrait permettre à notre revue de faire un saut qualitatif important, et il ne tient qu'à vous d'en faire aussi un saut quantitatif. Je compte sur vous.

Henri Métivier

Président du comité de la revue 